\title{
EL NIÑO DISLÉXICO Y SU ENTORNO EDUCATIVO. PERCEPCIONES Y REPRESENTACIONES SOBRE LA DISLEXIA ${ }^{1}$
}

\author{
THE DYSLEXIC CHILD AND HIS EDUCATIONAL ENVIRONMENT. \\ PERCEPTIONS AND REPRESENTATIONS ON THE DYSLEXIA
}

\author{
María Paula Antognazza \\ Universidad Católica del Uruguay, Uruguay \\ María del Luján González Tornaría \\ Universidad Católica del Uruguay, Uruguay
}

\begin{abstract}
Resumen: Se presenta un estudio sobre las percepciones y representaciones de las familias, docentes, terapeutas y niños sobre la dislexia, en el que se indaga en sus respectivas vivencias y necesidades. Se exponen el diseño y resultados hallados en la investigación de corte cualitativo, basado en las entrevistas realizadas al niño, sus padres, docente y terapeuta. Entre los principales resultados, se destaca que tanto la familia como las instituciones educativas dan una respuesta parcial a las dificultades de aprendizaje y no existe un proyecto integrador que sea estimulante, tanto para el niño disléxico como para su familia. Cuando se logra realizar un tratamiento especializado, los padres no se sienten involucrados. Se detecta ausencia de una visión sistémica de la dificultad, lo que implica pérdida de esfuerzo, energía y tiempo para todas las partes.

Se realizan algunas consideraciones respecto a la importancia de pensar especialmente las percepciones de los propios niños disléxicos, colocando tales asuntos en el centro de la orientación diagnóstica y terapéutica.
\end{abstract}

Palabras clave: dislexia, relación familia-escuela, modelo ecológico-sistémico.

\begin{abstract}
A study on the perceptions and representations of the dyslexic children's families, teachers and therapists is presented. The investigation is a result from a qualitative method of study, based on interview for the children, their parents, teachers and therapists. Our results show that families and educational centers give a partial answer to the learning difficulties and also evidence the lack of an integrated project that stimulates the dyslexic child and his family. When a specialized treatment is implemented, the parents do not usually feel involved. We have also found the lack of the systemic approach to this disorder, resulting in a inefficient use of energy and time by all actors involved.

We also consider the importance of taking in to account the dyslexic children's own perceptions; finally, in this direction is important to focus on such matters for therapy and assessment orientation.
\end{abstract}

Keywords: dyslexia, family-school relationship, ecological-systemic Model.

1 (N. de la Ed.). Esta comunicación presenta el trabajo realizado en el marco de la primera Memoria de Grado de la Licenciatura en Psicopedagogía de la Universidad Católica del Uruguay.

\section{INTRODUCCIÓN}

El objetivo del presente estudio consistió en indagar en las percepciones y representaciones del niño disléxico, los padres, docentes y terapeutas en relación a la dislexia.

Se considera importante, investigar cómo interactúan todos los actores que constituyen puntos de apoyo y de intervención, en la tarea común de educar a un niño con dificultad específica del aprendizaje.

La dislexia tiene una implicancia personal y social grave, ya que impide que se enriquezcan los patrones conceptuales y que la persona se integre a la sociedad como ciudadano responsable y consciente. (Cuadro, 2010).

Correspondencia: María Paula Antognazza. Colaboradora del Dpto. de Psicología del Desarrollo y Educación. Facultad de Psicología. Universidad Católica del Uruguay. Correo Electrónico: paula.antognazza@hotmail.com. 
La relevancia que tiene este problema entre los trastornos de aprendizaje, se basa en la magnitud de las limitaciones que ejerce sobre el rendimiento académico, en la medida que el lenguaje escrito es esencial para el desarrollo cognitivo, afectivo y social de las personas. Se habla de una prevalencia de un $5 \%$ a un $10 \%$ a nivel mundial (Mateos, 2009), mientras que en Uruguay se estima una prevalencia de un 15,4 $\%$. Esta cifra puede explicarse porque algunos individuos que se incluyen como disléxicos, son en realidad sujetos con retraso lector. (Cuadro, 2005; Cuadro, 2010).

Cabe destacar que la investigación que se llevó a cabo se encuentra enmarcada en diversos trabajos que se han ido realizando en el Departamento de Psicología del Desarrollo y Educación de la Facultad de Psicología de la Universidad Católica del Uruguay. Entre ellos se encuentra el de Cristóbal (2007), que realizó una investigación cualitativa acerca de la interacción entre el niño y su contexto desde la mirada de los padres. Da Silva (2006) profundizó en aspectos teóricos acerca de la familia, escuela, dificultades de aprendizaje e intervención psicopedagógica y planteó líneas orientadas a padres con hijos con problemas escolares, para que puedan vincularse con más éxito a la escuela a pesar de sus dificultades. A su vez, Delmás y Rivera (2007), propusieron una intervención con padres de adolescentes diagnosticados con una dificultad de aprendizaje o que presentan bajo rendimiento académico.

Para analizar esta temática, se considera como marco de referencia el modelo ecológicosistémico propuesto por Urie Bronfenbrenner (1987). El postulado básico de dicho modelo plantea por un lado que el desarrollo humano supone la progresiva acomodación mutua entre un ser humano activo, que está en proceso de desarrollo, y por otro, las propiedades cambiantes de los entornos inmediatos en los que vive esa persona. Esta acomodación mutua, se va produciendo mediante un proceso continuo y se ve afectado, por los vínculos que se establecen entre los diferentes entornos en los que participa la persona y los contextos más grandes en que esos entornos están incluidos.

La dislexia es una dificultad específica del aprendizaje, que al ser diagnosticada tiene su implicancia en la familia y en el entorno educativo. Por ello es importante considerar el contexto escolar, familiar y social, en la toma de decisiones, para generar cambios que hagan posible mejorar la situación frente a la cual nos encontremos. Tanto para la evaluación como para la intervención psicopedagógica de un niño, se deben tener en cuenta estos ámbitos, considerando fortalezas y debilidades, para armonizar y optimizar los resultados (Cuadro, 2010; Sánchez Cano y Bonals, 2005).

Se destaca que la relación familia-escuela, familia-terapeuta, escuela-terapeuta, es fundamental, pero no es fácil lograr una fórmula de alianza efectiva entre ellos.

En palabras de Bassedas, Huguet y Solé (2002, 325), "durante todo el desarrollo del niño, el conocimiento mutuo y el establecimiento de acuerdo entre el contexto familiar y el escolar actúan en beneficio del niño y promueven su bienestar". Los mismos autores enfatizan que para que se establezca una buena relación familia-escuela, deben basarse en el respeto mutuo, la confianza y la aceptación de las particularidades de cada una. Esta relación puede enriquecerse con aportes de otros profesionales como el psicopedagogo, encontrando maneras constructivas de trabajar en conjunto.

Según Huguet $(1999,129)$, "la relación con la escuela (inicio de la escolaridad, exigencias que plantea a la familia, valoraciones, conflictos), como sistema con el que tiene una continuada y estrecha relación, a menudo es fuente también de cambios y transformaciones en la organización y dinámica familiar, cambios que pueden ser vividos como adaptación y crecimiento o como conflicto y frustración, según la capacidad del niño y de la familia de adaptarse a la relación con ese contexto extrafamiliar".

Según Planas (2007), el colegio va a representar para el niño la adaptación a un contexto más amplio y complejo. En el pasaje de la familia a la escuela, se hace imprescindible la coordinación entre ambos contextos.

Desde las instituciones educativas, no se puede comprender al alumno sin tener presente la realidad familiar. La familia, tampoco puede dejar de lado el impacto que el entorno educativo provoca en el desarrollo de sus hijos. (Planas, 2007). En la literatura se insiste en la 
importancia de una visión orgánica y sistémica para el abordaje de la relación familia-centro educativo, especialmente cuando existen dificultades del aprendizaje (Dowling, 1996; Rodrigo y Palacios, 1998).

Por lo que venimos de plantear, existe en la actualidad, la tendencia a adherirse a modelos de colaboración entre familias y centros educativos, algunos de ellos involucrando a la comunidad. Los exponentes más relevantes de dichos modelos se describen en González, Vandemeulebroecke y Colpin (2001).

\section{OBJETIVO DEL ESTUDIO}

Con el propósito de profundizar en el entorno educativo del niño disléxico el objetivo general del estudio consistió en indagar sobre el proceso diagnóstico, terapéutico y educativo, analizando la interconexión del ámbito familiar, escolar y terapéutico.

Tal como lo expresan autores como Huguet (1999) y Planas (2007) es fundamental que el psicopedagogo conozca las percepciones que tienen las personas sobre las dificultades que les afectan, al punto que es una de las tareas que se le adjudican a este profesional en las instituciones educativas.

Rivera y Milicic (2006) han tomado también el estudio de las percepciones y representaciones de padres y profesores de enseñanza general básica como objetivo principal de estudio para el logro de alianzas efectivas entre las familias y escuelas.

De acuerdo a Rodrigo y Palacios (1998), abordar el estudio del niño disléxico y su entorno educativo, bajo esta perspectiva tan compleja, requiere un gran esfuerzo e ingenio metodológico en un doble plano: el de los procedimientos e instrumentos de recogida de la información y el de las técnicas a utilizar para realizar el análisis de los datos. Comprendiendo lo complejo de esta situación, se trata de lograr en este estudio un acercamiento a los distintos sistemas involucrados, para comprender su funcionamiento y la importancia de las relaciones interpersonales recíprocas.

\section{Método}

\subsection{Participantes}

Para este trabajo se seleccionaron cinco niños diagnosticados como disléxicos, de diferentes grupos etarios, diferentes grados escolares e insertos en distintas Instituciones educativas públicas. Se pretendió así que los casos fueran heterogéneos y que brindaran la mayor información posible, acerca de vivencias $y$ actitudes de los actores y del entorno en relación a la dislexia.

Las edades de los niños oscilaron entre 9 y 13 años, 3 de sexo masculino y 2 femenino, en niveles de escolaridad desde 4to de educación primaria a 2do año de educación secundaria. Participaron también sus padres, docentes y terapeutas.

Las familias de la población seleccionada, pertenecían a nivel socio-económico-cultural medio bajo.

En la selección, se tuvieron en cuenta los siguientes criterios: que fueran niños cuyo diagnóstico de dislexia datara de al menos un año, sin patologías o dificultades asociadas y con tratamiento en curso. Estos niños se reclutaron en un centro que mantiene un convenio con el Banco de Previsión Social ${ }^{1}$. En dicho centro se estaban desarrollando los tratamientos de los niños. A la hora de seleccionarlos, también se tuvo en cuenta la asiduidad y el compromiso de los niños y sus familias con los tratamientos.

\subsection{Instrumento para la recolección de datos}

Se realizaron entrevistas en profundidad a los niños disléxicos, padres, docentes y terapeutas. Este instrumento es considerado en las investigaciones de corte cualitativo, como una de las fuentes y métodos más sobresalientes para acceder a la información (Kazdin, 2001).

Tal como plantean León y Montero (2003) en la entrevista en profundidad, se pone énfasis en descubrir y describir lo particular, lo peculiar.

1 El Banco de Previsión Social (BPS) es el instituto de seguridad social estatal de Uruguay; coordina los servicios estatales de previsión social y organiza la seguridad social. Brinda prestaciones a niños con dificultades del aprendizaje y del desarrollo, entre otros. 
En la presente investigación, se utilizó una pauta de preguntas abiertas, siguiendo el propio desarrollo de la entrevista, tratando de lograr libertad, confianza y espontaneidad en los entrevistados, aceptando los aportes que ellos podían realizar.

Mediante estas entrevistas, se trató de establecer contactos directos y de mayor profundidad, con el fin de poder acceder a aspectos subjetivos vivenciados por los entrevistados. Para esto se procuró crear un clima de respeto, empatía y confidencialidad.

\subsection{Procedimiento}

Se contactó con la directora del centro particular dedicado al trabajo de atención a niños y adolescentes con dificultades de aprendizaje. En esta oportunidad, se firmó la carta de autorización para realizar la investigación en el centro. Una vez contactados los participantes del estudio, tomaron conocimiento de los objetivos del trabajo y se les presentó el consentimiento informado.

Se realizaron las entrevistas a cinco niños disléxicos, su familia, docente y terapeuta según los criterios expuestos anteriormente. Vale destacar que cada entrevista comenzó con una breve presentación verbal de la entrevistadora y de la investigación, mencionando su objetivo principal. Para el caso de este estudio, se realizó un total de 20 entrevistas cuya duración fue aproximadamente de 45 minutos cada una. Las mismas se llevaron a cabo entre los meses de julio y agosto del año 2010. Se realizó una transcripción fiel de las grabaciones para poder hacer un análisis exhaustivo del material.

Respetando los criterios propuestos por Bardin (1986) para realizar el análisis de contenido se siguió una técnica de interpretación de textos, de acuerdo al método científico. Se tuvieron en cuenta las cualidades que debe reunir un conjunto de categorías: homogéneas, exhaustivas, exclusivas, objetivas y adecuadas. El análisis de contenido propuesto por Bardin combina intrínsecamente, y de ahí su complejidad, la observación y producción de los datos, y la interpretación o análisis de los mismos.

Al tratarse de un estudio de tipo cualitativo, se establecieron, a partir del estudio de la lite- ratura (Mateos, 2009; Sánchez Cano y Bonals, 2005; Herrera y Delfior, 2005; Serrano, 2010; Cuadro, 2010), tres áreas para la delimitación de los temas, a saber: proceso diagnóstico, educativo y terapéutico, ya que estas son las etapas por las cuales atraviesan los niños y su entorno educativo al ser evaluados y tratados por alguna dificultad de aprendizaje Dentro de los mismos se incluyeron varias categorías a efectos de organizar la información recabada. Las categorías fueron predefinidas y fueron punto de partida para la construcción de las pautas de entrevista.

En el tema proceso diagnóstico se identificaron las siguientes categorías: demanda de evaluación diagnóstica; vivencia del diagnóstico; percepción, conocimiento y comprensión de la dislexia. En el tema proceso educativo se identificaron las siguientes categorías: metacognición; vinculación: docentes, compañeros; rendimiento académico; percepción de los distintos actores; relación familia-escuela y entidades del entorno comunitario. Respecto al último tema referido al proceso terapéutico las categorías fueron: plan de intervención del terapeuta; motivación del niño; percepción y valoración del tratamiento; percepción de la relación escuela-centro particular y expectativas respecto al futuro del niño.

Luego de realizar este análisis categorial, se expusieron los resultados y posteriormente se discutieron los mismos y se plantearon las reflexiones finales.

\section{PRESENTACIÓN Y DISCUSIÓN DE RESULTADOS}

Los cinco niños disléxicos seleccionados y diagnosticados por el centro particular que los atiende, tenían antecedentes de dislexia en su familia, lo cual creó la expectativa de que ellos y sus padres poseían algún conocimiento sobre este tema. Sin embargo, las familias manifestaron desconcierto frente a la dificultad de aprendizaje de sus hijos y no disimularon sentimientos encontrados de angustia y alivio, por saber que la dificultad al fin tenía un nombre, "dislexia".

Encontramos, tal como plantea Milicic (1988), que cuando el lenguaje técnico no 
es comprendido por la familia, se transforma en etiquetas vacías, sin significado o incluso, amenazantes. Se pudo apreciar en el estudio que los padres buscaron causas familiares justificativas dentro de la familia, generándose en ocasiones conflictos en la pareja, así como la sobreprotección del niño. Esto sucede cuando no hay aceptación del problema, siendo un desafío terapéutico interesante, cohesionar a ambos padres en una tarea común, fortaleciendo los vínculos entre ambos. Producto de su confusión, en cuanto al significado de la dificultad, los padres no tenían claro qué esperar del aprendizaje de sus hijos, del tratamiento, de la escuela y de su futuro desempeño como adultos. Esta confusión, nos hace pensar desde una mirada sistémica, cuál es el mensaje que recibe el niño por parte de sus padres, respecto a los logros que pueda alcanzar. El desconcierto de parte de los adultos respecto del trastorno puede impactar en las expectativas que se tengan en relación a la trayectoria escolar del niño y su futuro.

Sucedía también que en los casos entrevistados, más padres que maestros se dieron cuenta de la dificultad en los niños, a través de ciertas señales tales como: dificultades en la lectura, problemas de concentración, confusión de letras. Sin embargo los padres no recibieron ningún señalamiento de la institución educativa en la etapa pre-escolar ni escolar. Esto generó desconfianza de la familia frente a la institución educativa, surgiendo como causa de este hecho la posible falta de preparación de los docentes en cuanto a dificultades de aprendizaje. A su vez, esta deficiencia en la formación fue aceptada y confirmada también por los docentes entrevistados. Se observó la molestia de los padres frente a los comentarios que realizan algunos educadores al referirse a sus hijos simplemente como perezosos, sin tomar en cuenta la dificultad específica que presentan. Justamente Comellas (2009), advierte que la desconfianza entre la relación familia-escuela, genera un clima y una falta de comunicación que termina perjudicando el proceso educativo de los niños.

Siguiendo a Bronfenbrenner (1987), desde una perspectiva ecológica, se puede pensar que las características del encuentro entre familia-escuela, a nivel del mesosistema, al no ser óptima no colabora con el descenso de la ansiedad que genera, de por sí, esta dificultad específica en la familia.

Cabe destacar, el paralelismo que realizaba ya Tizard (1961, en Milicic 1986), al establecer una comparación entre las diferentes etapas de aceptación y elaboración del problema, de las familias con niños con Retardo Mental, con las etapas que viven las familias de niños con dificultades del aprendizaje. Esto se constata en las entrevistas realizadas en este trabajo, en las cuales se apreció, la toma de conciencia de que algo no estaba funcionando bien, el reconocimiento de la existencia de un problema en el niño, la búsqueda de causas a ese problema, la búsqueda de soluciones y finalmente, la aceptación de la dificultad. En los niños disléxicos se suma a estas etapas citadas, el desafío de entender por parte de los padres que sus hijos sin tener C.I. descendido, tengan dificultades de aprendizaje.

Surge del presente trabajo realizado que tanto los padres, como los terapeutas y docentes, consideraron explícitamente muy importante la coordinación entre ellos para aunar esfuerzos y así brindar la mejor atención al educando. Si bien todos estaban de acuerdo con la necesidad de una alianza, ésta no se hacía efectiva, al momento de cada uno asumir su responsabilidad de comunicación. Los adultos justificaron su ausencia de diálogo, por falta de tiempo responsabilizándose entre ellos. En general, en todos los casos, las comunicaciones quedaban limitadas a informes escritos que se intercambiaban entre las partes en momentos formalmente definidos.

Los maestros reclamaban más apoyo de los padres en las tareas domiciliarias, momento de angustia y tensión que en general no sabe encarar la familia.

Por otra parte, se menciona lo difícil que es trabajar en red y tener en cuenta otras instituciones del ámbito comunitario. Varios padres y maestros afirmaban, que la relación entre las diferentes instituciones tiene que mejorar, pero no es más que una aspiración que no se concreta en la práctica educativa.

Parece claro que estos desencuentros se resolverían mediante una distribución clara de responsabilidades, que quede plasmada en 
compromisos explícitos, a efectos de elaborar planes de trabajo integrados.

Bronfenbrenner (1987) afirma que el consenso de metas entre entornos y un creciente equilibrio de poderes entre ellos, redundan en bien de la persona en desarrollo. Siguiendo el pensamiento antes mencionado, destacamos que en las entrevistas se vislumbraba un equilibrio de poderes. Constatamos el beneplácito y aprobación de los padres que pudieron dar atención particular a sus hijos a través del BPS, a pesar del sinuoso camino burocrático recorrido para lograr la atención especializada. Los maestros dieron cuenta de su tranquilidad porque los alumnos disléxicos eran atendidos y los terapeutas cumplieron con su rol del tratamiento específico de la dificultad. De todos modos, el consenso del cual habla el autor antes mencionado, es difícil de lograr, aún cuando las relaciones entre los diferentes profesionales de las distintas instituciones sean correctas y armoniosas y haya ánimo y predisposición para el encuentro. Como las instituciones trabajan de forma aislada, las relaciones interpersonales se dan con escaso nivel de diálogo.

El problema radica tal vez, en que muchas intervenciones pierden de vista el objetivo básico que es el niño disléxico, quien manifestó en las entrevistas realizadas para este trabajo, ser depositario de una educación que no comprende y le es distante.

\section{CONCLUSIONES}

Repasando el objetivo planteado en este estudio, pudimos comprobar que el proceso diagnóstico y las vivencias que despierta especialmente en el niño y sus familias son clave para la evolución del caso. Aspectos como la comprensión de lo que significa la dislexia y cómo abordarla pueden constituir, de no ser correctamente trabajados, la primera fuente de desencuentro e impactar negativamente, impidiendo que en el proceso educativo y en el tratamiento se haga uso de los recursos que tienen todas las partes.

En los cinco casos los padres transitaron por un periplo de instituciones y profesionales para llegar al diagnóstico y luego a un centro de tratamiento. Tuvieron que enfrentar la situación y apoyar al niño, sin lograr muchas veces que esta actividad fuera gratificante. Los padres no se sintieron orientados para acompañar a sus hijos en el entorno familiar. Asimismo, los maestros en la escuela no se involucraron con los terapeutas y principalmente recibieron informes de evolución, sin poder integrar indicaciones a su tarea diaria.

En cuanto a las vivencias captadas en las entrevistas de los niños involucrados, observamos a través de sus palabras cierto retraimiento, falta de información sobre su dificultad específica, poca espontaneidad, falta de fluidez en su expresión, cumpliendo tareas por obligación sin el placer de aprender, y poco entusiasmo en sus actividades. No lograban la metacognición y no podían explicar en qué consistía su dificultad, siendo esto tan importante para clarificar expectativas y para obtener buenos resultados en el tratamiento. Las intervenciones eran vividas como muy largas, algunos niños pensaban que en esas horas podrían dedicarse a otras actividades. Sin embargo, los terapeutas sentían que había que aumentar las sesiones de intervención psicopedagógica para que el tratamiento fuera más efectivo. Llama la atención que quien es el centro de nuestras preocupaciones, "el propio niño disléxico", sea quien menos información posea sobre su dificultad y evolución.

En las entrevistas realizadas, los terapeutas responsables de los tratamientos en el centro particular, son maestros especializados y fonoaudiólogos. No aparece mencionado en los equipos el Licenciado en Psicopedagogía en cuyo perfil se encuentra la atención de esta dificultad específica, siendo este profesional de indudable importancia para la integración de equipos interdisciplinarios y para ser tenido en cuenta en la elaboración de políticas públicas.

Los docentes, por su parte, se encuentran desbordados y desvalidos para el trabajo con estos niños, aduciendo en general falta de formación básica y falta de orientación y conexión con los equipos tratantes.

Es fundamental entonces que el tratamiento de esta dificultad específica esté previsto desde el proyecto de las instituciones, para no dejar librado a las personas y a sus disponibilidades particulares, la detección y el tratamiento de las mismas. Máxime cuando la realidad social 
y educativa del Uruguay, especialmente con los aprendizajes del lenguaje escrito en los sectores con privación sociocultural, requiere respuestas efectivas tal como lo especifica Cuadro (2010).

En síntesis, surge la necesidad de buscar y encontrar una mirada integradora, de ida y vuelta, desde las instituciones involucradas; la familia, el centro educativo y centro de tratamiento, teniendo siempre presente como objetivo, optimizar la atención del niño con dificultad específica. Percepciones y representaciones de quien es objeto de este estudio, deberían ser tenidos en cuenta como brújula de orientación diagnóstica y terapéutica. Destacamos la contribución del Licenciado en Psicopedagogía en el rol articulador con las Instituciones del entorno educativo del niño con dificultades de aprendizaje.

Al finalizar este trabajo, sugerimos algunos temas que consideramos de interés para futuras investigaciones tales como:

- Indagar si la dinámica observada en el presente trabajo es similar en niños disléxicos de estratos sociales más elevados, que pueden tener acceso a otros servicios y contar con equipos en los centros educativos a los que concurren.

- Profundizar en la orientación a los padres del niño disléxico para que el apoyo del hogar sea gratificante y redunde en un mejor rendimiento del niño.

- Estudiar la trayectoria escolar de los niños disléxicos y su vivencia sobre la educación formal.

- Identificar si existen proyectos institucionales en la Enseñanza formal que tengan en cuenta el tratamiento de esta dificultad, haciendo uso al máximo de los recursos de todas las partes.

\section{REFERENCIAS}

Bardin, L. (1986). El análisis de contenido. España: Ediciones Akal.

Bassedas, E. Huguet, T. Solé, I. (2002). Aprendery enseñar en educación infantil ( $3^{\circ} \mathrm{ed}$.). Barcelona: Editorial Graó.

Borrell, S. (2007). La participación de las familias en la escuela. Revista Española de Pedagogía, año LXV, $N^{\circ} 238,559-574$.

Bravo Valdivieso, L. Milicic, N. Cuadro, A. Mejia, L. Eslava, J. (2009). Trastorno del aprendizaje: investigaciones psicológicas y psicopedagógicas en diversos países de Sud América. Ciencias Psicológicas, III (2): 203-218.

Bronfenbrenner, U. (1987). La ecología del desarrollo humano. Buenos Aires: Paidós.

Comellas, M. J. (2009). Familia y escuela: compartir la educación. Barcelona: Editorial Graó.

Cristóbal, M (2007). El niño disléxico y su familia. La interacción entre el niño y su ambiente ecológico desde la mirada de los padres. Memoria de grado. Licenciatura en Psicología. Facultad de Psicología. Universidad Católica del Uruguay.

Cuadro A. (2010). La lectura y sus dificultades: la dislexia evolutiva. Montevideo, Uruguay: Editorial Grupo Magro.

Cuadro, A. (2005). Características de los malos lectores en relación a los buenos lectores en niños uruguayos. Tesis Doctoral. Universidad de Murcia, España.

Da Silva, S. (2006). Familia, Escuela, Dificultades de Aprendizaje. Intervención

Delmás, F. \& Rivera, M. (2007). La familia del niño y del adolescente con dificultades de aprendizaje. Trabajo Final de Especializaciòn en Psicología Educacional. Facultad de Psicología: Universidad Católica del Uruguay.

Dowling, E. (1996): Marco teórico: Un enfoque sistémico conjunto de los problemas educativos con niños. En: Dowling E. y Osborne E. Familia y escuela. Una aproximación conjunta y sistémica a los problemas infantiles. Barcelona: Paidós. (21-50).

González Tornaría, M.L.; Vandemeulebroecke, L. y Colpin, H. (2001): Pedagogía Familiar. Aportes desde la teoría y la investigación. Montevideo: Trilce.

Herrera, L. y Defior, S. (2005): Una aproximación al procesamiento fonológico de los niños pre lectores: conciencia fonológica, memoria verbal a corto plazo y denominación. Psyke, 14, (2)

Huguet, T. (1999). El asesoramiento psicopedagógico y la colaboración entre la familia y el centro educativo. En: Monereo y Solé (1999). (coord.) El asesoramiento psicopedagógico: una perspectiva profesional y constructiva. Madrid: Alianza Editorial. (127-148).

Instituto Nacional de Estadística. (2010). Estimación de la pobreza por el método del ingreso. Recuperado el 2/V/2011 de http://www.ine.gub.uy/biblioteca/estimacion_pobreza_2010

Kazdin, A. (2001). Métodos de investigación cualitativa. En: Kazdin, A. Métodos de investigación en psicología clínica. (3ªd.) México: Prentice Hall, pp.245-262.

León, O. Montero, I. (2003). Métodos de investigación en psicología y educación. (3a.Ed.). España: Mc Graw-Hill.

Mateos, R. (2009). Dificultades de aprendizaje. Psicología educativa; 15 (1): 13-19. 
Milicic, N. (1986). Algunos modelos de evaluación familiar y su implicación para la intervención en familias de niños con dificultades para aprender. Ponencia. VIII Jornadas de las Sociedad de Dislexia del Uruguay. Libro de Actas pp. 21-23. Montevideo.

Milicic, N. (1988). La familia y el proceso de diagnóstico. Revista de Orientación educacional. V (2/3), 41-59.

Planas, M. (2007). Familia y escuela: estrategias para una relación constructiva. En: Sánchez Cano, M. Bonals, J (2007). (coord.) Manual de asesoramiento psicopedagógico. Barcelona: Editorial Graó. (203-230).

Rivera, M. Milicic, N. (2006). Alianza Familia-Escuela: Percepciones, creencias, expectativas y aspiraciones de padres y profesores de enseñanza general básica. Psykhe; 15 (1): 119-135.
Rodrigo, M. Palacios, J. (1998) Familia y desarrollo humano. Madrid: Alianza Editorial.

Sánchez Cano, M. Bonals, J. (2005). La Evaluación Psicopedagógica. Barcelona: Graó.

Serrano, F. (2010). Dislexia: precisión y fluidez. Implicaciones para su detección y tratamiento. Comunicación personal. Facultad de Psicología. Universidad Católica del Uruguay.

Uruguay en PISA (2010). Primeros resultados en Ciencias, Matemática y Lectura del Programa Internacional de Evaluación de Estudiantes. Informe Ejecutivo. Recuperado el 21 de marzo de 2011 http://www. espectador.com/documentos/pisa2009_uruguay_informe_ejecutivo.pdf

Para citar este artículo:

Antognazza, M. P. \& González Tornaría, M. L. (2011). El niño disléxico y su entorno educativo. Percepciones y representaciones sobre la dislexia. Ciencias Psicológicas V (2): 163-200. 